\title{
LA (RE)PRODUCCIÓN DEL CAPITAL Y EL IMPACTO EN EL SUELO URBANO. EL CASO BARRIO GÜEMES, CÓRDOBA (ARGENTINA)
}

\section{THE (RE)PRODUCTION OF CAPITAL AND THE IMPACT ON URBAN LAND. THE CASE OF THE GÜEMES NEIGHBORHOOD, CÓRDOBA (ARGENTINA).}

\section{Ailen Suyai Pereyra ${ }^{1}$}

\section{RESUMEN}

La tendencia a escala global es la renovación urbana de espacios considerados en degradación, obsoletos o sin función alguna. A escala local esto se replica mediante la aplicación de un paquete de políticas públicas que intervienen en la producción de suelo urbano. El siguiente artículo presenta una investigación llevada a cabo en el barrio Güemes de la Ciudad de Córdoba entre los años 2000 y 2016. El objetivo es mostrar cómo la modificación del corpus normativo y la aparición de edificación en altu ra operan como elementos sensibles en la valorización del suelo urbano. Desde una perspectiva cualitativa se propone un conjunto de técnicas articuladas que evidencian la dinámica barrial. Se parte con un análisis documental de las políticas del Estado en todos sus niveles, su relación con el mercado urbano particularmente en barrio Güemes. A colación, se identifican y analizan las transformaciones urbanas que han sucedido en el período 2000-2016 como: 1) cambios normativos en relación a la zonificación y áreas especiales, 2) inmuebles con valor patrimonial, 3) nuevos negocios habilitados y 4) valorización del suelo urbano. Los resultados exponen que las políticas de renovación urbana desencadenaron una dinamización de actividades modificando el uso y un incremento del valor del suelo.

Palabras clave: suelo urbano, barrio Güemes, políticas urbanas, valorización, corpus normativo.

\section{ABSTRACT}

The trend on a global scale is the urban renewal of spaces considered degraded, obsolete or without any function. At the local level, this is replicated through the application of a package of public policies that intervene in the production of urban land. The following article presents an investigation carried

\footnotetext{
${ }^{1}$ Doctora en Estudios Urbano Regionales por la Universidad Nacional de Córdoba y la Bauhaus Universität Weimar (Alemania). Geógrafa de la Facultad de Filosofía y Humanidades de la Universidad Nacional de Córdoba. Becaria Posdoctoral del Consejo Nacional de Investigaciones Científicas y Tecnológicas (CONICET) con lugar de trabajo en el Instituto de Investigación de Vivienda y Hábitat (INVIHAB). Miembro del Programa de Investigación de Ideología y Prácticas Sociales en conflicto (CIECS-CONICET). Docente colaboradora del Seminario Ciudad y producción histórica de las subjetividades desde una perspectiva crítica de la Facultad de Psicología (UNC). Docente del nivel medio. Afiliación: Instituto de Investigación de Vivienda y Hábitat (INVIHAB). Argentina. ORCID: https://orcid.org/0000-0003-1796-4271 Becaria Posdoctoral CONICETE-mail: ailen.suyai.pereyra@unc.edu.ar
} 
out in the Güemes neighborhood of the City of Córdoba between 2000 and 2016. The objective is to show how the modification of the normative corpus and the appearance of high-rise buildings operate as sensitive elements in the valorization of the urban land. From a qualitative perspective, a set of articulated techniques is proposed that evince the neighborhood dynamics. It starts with a documentary analysis of State policies at all levels, its relationship with the urban market, particularly in the Güemes neighborhood. In addition, the urban transformations that have occurred in the period 2000-2016 are identified and analyzed as: 1) regulatory changes in relation to zoning and special areas, 2) properties with patrimonial value, 3) new enabled businesses and 4) valuation of urban land. The results expound the urban renewal policies that triggered a dynamization of activities by modifying the use of land and an increase in the value of land.

Keywords: urban land, Güemes neighborhood, public policies, valuation, normative corpus.

\section{INTRODUCCIÓN}

En este artículo se presentan los resultados de investigación sobre el barrio Güemes de la ciudad de Córdoba (Argentina) entre los años 2000 y 2016. Se considera que desde los 2000 el barrio asiste a un proceso de crecimiento inusitado. En un sector del mismo, la feria histórica artesanal aumentó la cantidad de artesanos y la variedad de productos ofrecidos, a la par emergieron los comercios que forman parte de la oferta comercial, gastronómica y cultural del barrio. Hace varios años, presenta nuevos actores económicos que se pueden observar en la apertura de galerías comerciales; ubicadas sobre el eje de las calles Belgrano, Achával Rodríguez, Fructuoso Rivera. Estos se dedican a la venta y exposición de piezas de arte, gastronomía, negocios de diseñadores cordobeses, entre otros. En el plano físico-arquitectónico se desarrolló la idea del "desarrollo sustentable" en la intervención de los techos de las galerías y paneles de energía solar en conjunto con la creciente aparición de edificaciones alrededor de la feria artesanal histórica.

Como hipótesis de trabajo se entiende que la modificación del corpus normativo, la aparición de edificación en altura y el boom económico se tradujo en el territorio a través de la valorización del suelo urbano y la retroalimentación en el espacio con el emplazamiento de nuevas actividades comerciales y servicios culturales. A la par, en el espacio barrial se presentan nuevos residentes con otros hábitos y prácticas que ponen en disputa los modos de habitar en el espacio.

El barrio Güemes colinda con el centro de la ciudad, surgió como asentamiento sin planificación alrededor de 1850. Una de las características principales es la heterogeneidad a su interior, pues se pueden identificar sectores que desde fines del siglo XIX están presentes en él. 
También es considerado un "barrio tradicional" por la Municipalidad de Córdoba debido a la historia barrial y por albergar lugares emblemáticos para la ciudadanía cordobesa. Como ya se adelantó, durante los fines de semana y feriados alberga la feria histórica de entre 600-700 artesanos que permiten la concurrencia de aproximadamente 10.000 personas. A modo de paseo completo, en la proximidad de dicha feria se encuentran bares, restaurantes y espacios con propuesta artística que atraen a un público visitante.

El artículo se inscribe dentro de la perspectiva del materialismo histórico, enriquecido con otros autores. Para ello se desarrollarán conceptos claves como el de transformaciones urbanas (revolución urbana, los ajustes espacio temporales) desde el neomarxismo propuesto por Harvey. En esa línea, se concibe al espacio como una construcción social (LEFEBVRE, 1974), en el cual a modo de palimpsesto encontramos la triada espacial propuesta por Lefebvre, ellos son los espacios: concebidos, percibidos y vividos ${ }^{2}$ (LEFEBVRE, 1974; SOJA 2000). En dicho espacio existen múltiples actores y operan a diferentes escalas, con intereses particulares.

Desde hace unos años, la evidencia indica que los espacios elegidos por los municipios y para la intervención pública y/o privada son los barrios pericentrales, puesto que son espacios cercanos al centro y considerados estratégicos. Con el argumento de renovar, rehabilitar y valorizar zonas poco aprovechadas o degradadas se ponen en marcha proyectos de renovación urbana, con el objetivo de mejorar la calidad de vida de la población y dinamizar el sector (BRITES, 2017). A riesgo de simplificar, estas transformaciones fueron producto de los cambios político-ideológicos, de los modelos e instrumentos de gestión urbana puestos en juego en los diversos momentos históricos y de las propias prácticas sociales y culturales de los habitantes.

Esta investigación propone una estrategia metodológica cualitativa, mediante la implementación de diferentes técnicas de recolección, selección y análisis de la información obtenida. Por ello, en primer lugar, se realiza una descripción y análisis documental de las políticas del Estado en todos sus niveles, su relación con el mercado urbano particularmente en barrio Güemes. A colación, se identifican y analizan las transformaciones urbanas que han sucedido en el período 2000-2016 como: 1) cambios normativos en relación a la zonificación y áreas especiales, 2) inmuebles de diferente valor patrimonial, 3) nuevos negocios habilitados y 4) valorización del suelo urbano barrial. De esta manera, se centrará la mirada analítica en las transformaciones urbanas del barrio Güemes, en el marco de la metamorfosis del espacio urbano que conjugó un proceso de intersección y asociatividad entre políticas públicas y expansión inmobiliaria.

\footnotetext{
${ }^{2}$ La triada conceptual está compuesta por: el espacio percibido: la experiencia que implica la producción y reproducción. El espacio concebido: representado por los planificadores, urbanistas y tecnócratas. El espacio vivido: en él habitan los pobladores, ellos experimentan y modifican el espacio dominado (LEFEBVRE, 1974).
} 


\section{LA PRODUCCIÓN DE ESPACIO URBANO DENTRO DEL MODO DE ACUMULACIÓN CAPITALISTA}

Diversos autores que abordan la cuestión urbana han señalado que a las ciudades y/o algunos sectores urbanos se los exhibe como una mercancía más, la cual debe atraer, mantener y competir por nuevas inversiones (JACOBS, 1973; VAINER, 2000; ZUKIN, 2010). Este fenómeno se inscribe en las recurrentes y sucesivas fases de sobreproducción, sobreinversión y sobre endeudamiento del sistema capitalista. De esta manera, se someten las urbes a cambios estructurales, a un conjunto de transformaciones que dan lugar a una supuesta nueva solución espacial, esto se denominó reestructuración económica (MÉNDEZ y CARAVACA, 1996).

Siguiendo a Wallerstein (2005) las mencionadas fases no son otra cosa que las contradicciones inherentes al sistema, en esa dirección Harvey $(2004 ; 2011)$ afirmó que ante una crisis de acumulación, el sistema implementa ajustes espacio temporales con el objetivo de continuar con su reproducción. Es decir, el excedente de capital y dinero puede ser absorbido de tres formas: 1) a través del desplazamiento temporal de las "inversiones de capital en proyectos de largo plazo o gastos sociales (tales como educación e investigación)" (HARVEY, 2004 p.100); 2) por otro lado, el desplazamiento hacia nuevos mercados, otros espacios, nuevos recursos y nuevas formas de producir y/o 3) la combinación de estas dos opciones.

Ambos autores consideraron que muchas de las crisis tienen su origen en la especulación, en la presión que se ejerce por ejemplo, en los bienes inmuebles, y se escapan del control las instituciones financieras y bancos. El capital se expande por el globo y desplaza temporalmente para resolver dichas crisis y a su paso genera un "paisaje físico a su propia imagen y semejanza en un momento, para destruirlo luego" (HARVEY, 2004 p.103), esto lo que Harvey denominó destrucción creativa y tiene consecuencias tanto en el paisaje físico como en lo social. Dicho en otras palabras, el capitalismo toma sectores rentables para la producción y va captando todas las esferas de la vida para devolverlas como mercancías. La inmanente reproducción en los espacios urbanos implica la desposesión de todo lo material e inmaterial (lazos y tejido social, identidad, la esencia) de un lugar para luego tomarlo e incorporarlo a la línea de servicios y/o consumo. Una forma de plasmarse en el espacio es con la implementación de proyectos de renovación o revitalización urbana, cambios en la tipología de viviendas y perfiles edilicios con el objetivo de atraer inversiones. La acumulación de capital implica expandirse en el espacio y la consiguiente "destrucción creativa" (HARVEY, 2008) en zonas potencialmente rentables. Ahora bien, los espacios singulares o únicos (por su historia, arquitectura patrimonial, aglomeración comercial, servicios culturales, etc.) se ofrecen al mercado, de esta manera compiten por la llegada de un inversor. 
Por lo general, el Estado facilita la inversión privada e interviene mediante la ejecución de planes a través de alianzas público-privadas. En consecuencia, la lógica mercantil se instala y fomenta la privatización de espacios o de determinados bienes. También consolida las diferencias entre sectores en cuanto a la provisión de servicios públicos, la valorización del suelo y hacia las poblaciones que habitan estos lugares.

\section{EL ESTADO NEOLIBERAL}

Hacia la década de los setenta en un contexto de crisis económica el Estado Capitalista modificó sus objetivos y su funcionamiento. La crisis por la baja rentabilidad de las industrias de producción masiva y las contradicciones propias del Estado de Bienestar Keynesiano anunciaron su fin. Dentro de estos procesos, las transformaciones urbanas en el neoliberalismo realmente existente (THEODORE, PECK y BRENNER, 2009) están sujetas a la lógica capitalista, es decir a las "interacciones dependientes de la trayectoria y contextualmente específicas que se dan entre los escenarios regulatorios heredados (...) y proyectos emergentes de reformas neoliberales orientados al mercado" (THEODORE, PECK y BRENNER, 2009 p.3). En este nuevo marco de acumulación, los Estados comenzaron a barajar estrategias competitivas para fomentar la innovación, el emprendimiento y atraer inversiones. Como arquetipo se postuló un nuevo Estado de carácter competitivo (PECK, 2012) caracterizado por la subordinación de las políticas de gestión empresarial (HARVEY, 2013; 2008). El régimen del Estado supuso una reconfiguración de sus prioridades y jerarquías sensibles a la continua reproducción del capital. A escala global el escenario se caracterizó por el incremento de la competencia, la adaptación y diversificación de la producción y por lo tanto, de los trabajadores en torno a esta nueva lógica. El paradigma de la reestructuración económica se cristalizó en un nuevo tipo de Estado destinado a promover las condiciones económicas y extraeconómicas necesarias para el régimen de acumulación posfordista. Al respecto QUEVEDO mencionó:

El neoliberalismo sustentó una política económica donde su programa de gobierno era presentado no sólo como el más deseable sino como el único posible a partir de la configuración de axiomas teórico-explicativos y tópicos sacralizados por el saber experto. Su característica más importante es quizás pensar que lo real era programable mientras que lo social era una arena de efectos posibles desde una idea de libertad individual como epítome del libre mercado (QUEVEDO, 2018 p.138).

Esta nueva configuración económica de la década de los setenta, transformó los paisajes urbanos y a su vez, se modificaron los roles de los actores presentes en el mismo. Las ciudades se 
terminaron por consolidar como los lugares estratégicos que dan lugar al avance de proyectos reestructuradores neoliberales, pues son los escenarios privilegiados para la inversión de capital y el incremento de la renta económica (HARVEY, 2011).

En este contexto surgen los planes estratégicos como la revitalización de Bilbao Metropolitano en el norte de España, impulsado por Bilbao Metropoli-30. En América Latina, el primer Plan Estratégico fue ejecutado en la ciudad de Córdoba (1991-1995) basado en las experiencias europeas (FALÚ y MARENGO, 2002). Cabe destacar que las áreas cómo Bilbao, un sector de Barcelona (Plan 22@ Barcelona) e incluso Córdoba fueron elegidas gracias a su localización, el buen posicionamiento en los mercados frente a las preferencias de la demanda. Estos factores habilitarían captar un flujo continuo de inversiones para la supuesta recuperación, transformación y ampliación de modernos proyectos arquitectónicos. Los fundamentos esgrimidos eran amoldar los usos, una atribuida obsolescencia física y funcional de los edificios, de las infraestructuras y de los espacios públicos. También, la falta de niveles de servicio que no habían respondido a las necesidades de la población.

Ahora bien, la actualización de usos y las acciones públicas permitieron el mejoramiento tanto de los espacios como de los servicios y que las áreas crecieran en consonancia al ritmo global. A la par, en otras partes de la ciudad, la "falta" de interés por el Estado y otros actores dio lugar a una paulatina degradación y una devaluación global del área. Bajo un contexto neoliberal estas transformaciones forman parte de un proyecto de ciudad, es decir, la gestión empresarial (HARVEY, 2013) funciona como herramienta para la intervención de gobiernos locales junto al sector privado. Además que, muchas veces los mecanismos de participación ciudadana se constituyen como una pieza del plan pero sin una verdadera implicación en la planificación de espacios.

\section{TRANSFORMACIONES ESPACIALES A TRAVÉS DE PROCESOS DE RENOVACIÓN URBANA}

Los cambios materializados en algunos territorio, tanto en ciudades latinoamericanas (Buenos Aires, Salvador de Bahía en Brasil, México Distrito Federal, etc.) como en otras partes del mundo (New Orleans en los Estados Unidos, el distrito de Kreuzberg- Friedrichshain en Berlín, el puerto de Hamburgo en Alemania, etc.) demuestran cómo ciertos lugares se van transformado acorde al modo de reproducción capitalista. Pues, se trataba de áreas que en algún momento cumplieron funciones económicas-sociales jerarquizadas y luego por la dinámica misma del capitalismo, la sobreacumulación, dejan de ser rentables y pasan a ser espacios "obsoletos". Al respecto, la omisión de acciones públicas y/o privadas, la desatención y el crecimiento de situaciones sociales conflictivas (delitos, inseguridad, degradación) en estos sectores, funciona como argumento para que los gobiernos locales comiencen a planear el futuro y modernizarlos. 
Ahora bien, históricamente existieron procesos urbanos que desplazaron a población pobre hacia las periferias. El ejemplo más claro fue el de París con la intervención del Barón Haussmann, la apertura de grandes boulevares, la eliminación de un tipo de construcción precaria, la modificación del tejido urbano original y a la par una relocalización de la población original (ENGELS, 1873; BENJAMIN, 1999). La obra tuvo como finalidad mejorar la comunicación, las condiciones de higiene, seguridad, comodidades para hacer compras y facilitar el tránsito. También, estableció alturas uniformes de edificios, creó puntos de referencia como el Arc de Triomphe (Arco del Triunfo) y el Gran Palacio de la Ópera. Esta fuerte actuación en el centro de París tuvo como propósito preparar a la ciudad ante la llegada del conflicto. En el caso de presentarse una situación problemática, las fuerzas de seguridad atravesarían las grandes avenidas y rápidamente terminarían con las revueltas.

A escala latinoamericana las ciudades, desde 1990 fueron objeto de intervención para las políticas públicas urbanas. Algunos de los rasgos comunes que presentaron: inversión focalizada en áreas urbanas; modificación o nuevos marcos regulatorios del suelo urbano; la implementación de proyectos y/planes orientados a embellecer estratégicamente ciertos sectores (BOITO y ESPOZ, 2014) y/o la promoción del turismo (HERNÁNDEZ LÓPEZ, 2009) y zonas accesibles para emprendimientos con uso mixto del suelo (comercial y residencial). En ese sentido, la intervención se realiza en un barrio o en determinados sectores, posicionándolos como un laboratorio del modelo o arquetipo de ciudad que se pretende instalar. La renovación de áreas centrales y pericentrales desde hace varios años es una tendencia alrededor del mundo. Su elección se basa en la cercanía geográfica al centro comercial y administrativo, la variada oferta comercial, la posibilidad de transporte y conexión con otros sectores de la ciudad, la calidad de los servicios urbanos y el valor afectivo e histórico de la zona.

Las políticas urbanas que operan a través de acciones de renovación o rehabilitación para dinamizar económicamente determinados sectores alcanzó diferentes matices en los núcleos latinoamericanos. Este proceso operó como mecanismo de regulación de asentamientos informales, creación de áreas especiales y la modificación del corpus normativo, el impulso de inversión y revalorización de suelo urbano, el remozamiento del paisaje, como promotor del desarrollo comercial y turístico (BRITES, 2017). Dos elementos discursivos aparecen como posibilitadores del proceso de renovación urbana: el turismo y el patrimonio. En ese marco, bajo la recuperación patrimonial de ciertos lugares se dinamizan los territorios, por lo que el turismo se vuelve una herramienta económica que produce un excedente de plusvalía. La puesta en valor de bienes tangibles e intangibles atrae la afluencia de visitantes y, a la vez, que es rentable económicamente.

La transformación se expresa con una doble cara, por un lado el mejoramiento a zonas consideradas deprimidas, en desuso o abandonadas y la activación económica del sector (MARENGO, 2010; FALÚ y MARENGO, 2002). Por otro lado, las políticas neoliberales afectan directamente el 
mercado de suelo y la inversión inmobiliaria sea de uso residencial o comercial (JARAMILLO, 2009). La práctica discursiva también suele denominarlo "mejoramiento, renovación, reciclaje" tipificaciones que justifican las transformaciones en pos de generar una mayor rentabilidad económica. Sin embargo, muchas veces los proyectos tienen en cuenta las variables morfológicas y físicas, dejando en un segundo plano el impacto en el espacio próximo y las relaciones entre los habitantes con su territorio. Como consecuencia permite el afianzamiento de las diferencias entre clases sociales y de la fragmentación espacial.

\section{LA IMPLICANCIA DE LA TENDENCIA MERCANTILIZADORA EN EL SUELO URBANO}

Siguiendo a Arreortua Salinas (2019), para entender las transformaciones urbanas hay que ir más allá del fenómeno físico y buscar conocer la esencia del mismo“¿qué es lo que les da la explicación a estos procesos?" (ARREORTUA SALINAS, 2019 p.47). Se refiere, a la apariencia, al mejoramiento físico y además, a reflexionar sobre "la transformación social, es decir la transformación de las relaciones que existían en este espacio que ya no existen" (ARREORTUA SALINAS, 2019 p.48).

La intervención en la ciudad, la valorización de inmueble y el auge de algunas áreas y la degradación de otras, se plasman en el espacio como mayor segregación y el fenómeno de la gentrificación (GOTHAM, 2004). Si bien, esta categoría surgió para casos europeos y/o anglosajones como producto del desplazamiento de población original o trabajadores por burgueses, Glass (1964) con ciertos matices observó dicho fenómeno en las ciudades latinoamericanas. En estas, el proceso se refiere a una sustitución social y/o habitacional por una lógica basada en la rentabilidad.

La reestructuración espacial empuja a la población de ingresos medios y altos a elegir áreas centrales o tradicionales y ocuparlas relegando a las clases populares. Retomando la noción de gentrificación, muchos han sido los autores que han investigado sobre ella, le designaron con otros términos este proceso y aportando al debate. El término "ennoblecimiento" acuñado por Zukin (1996) y Carman (2011) categoría analítica que trató de castellanizar la palabra, hace referencia no solamente a la llegada de una clase social media-alta a un barrio y al desplazamiento de población originaria, sino al cambio de sentido del barrio, la transformación comercial que invita a usuarios solventes a consumir de él. Se trata de un proceso de apropiación cultural que "redefine el significado social de un lugar específicamente histórico para un segmento del mercado" (ZUKIN, 1996 p.87). Cada vez más intensamente los espacios, tienen un objetivo mercantil, intercambio de mercancías o de un paquete de experiencias para el disfrute.

Con la llegada de otra clase social a las áreas elegidas, las demandas de tipos de servicios se modifican. Surgen servicios personales (tales como spa, gimnasios) asequibles para elevados ingresos. 
Arreortua Salinas, basándose en los estudios de Florida (2010), denomina a esta población "nueva clase media" o "clase creativa":

están buscando espacios públicos adecuados, buscan centros de entretenimiento, buscan museos, teatros, cines; todo un estilo de vida que está en relación con su capital cultural y económico y por eso es que se genera esta inversión de capital privado (ARREORTUA SALINAS, 2019 p.52).

En esa línea, Galleguillos Araya-Schübelin y Robles Martínez (2018) consideran que en las sociedades post-industriales, la creatividad funciona como "un medio para mantener su hegemonía económica, transformando el conocimiento en una mercancía" (p. 3). Dos factores se vuelven motor de desarrollo económico: la cultura y la generación de una "imagen de ciudad". La "ciudad creativa" emerge como posibilitador para atraer trabajadores asociados al ámbito cultural, recreativo y tecnológico, estos forman aquello que denomina Florida (2010) como "clase creativa". Esta clase se siente atraída por lo económico, cuestiones referidas a su estilo de vida como los servicios personales o por lugares auténticos. Ciertamente, los espacio urbanos se adaptan a las necesidades de esta nueva clase creativa y con el supuesto de mejorar su posición económica. Esta dinámica impacta en los espacios y puede contribuir al proceso de gentrificación. En cuanto a los efectos positivos, Galleguillos Araya-Schübelin y Robles Martínez mencionaron que:

Al año 2011, las exportaciones mundiales de bienes y servicios creativos alcanzaban USD\$646 mil millones, y el $14 \%$ de éstas se originó en las Américas. A nivel mundial entre el 2002 y 2011 la exportación de bienes y servicios creativos creció un $134 \%$ y son el quinto rubro en el mundo a nivel de ventas. En Latinoamérica y el caribe las exportaciones de este sector alcanzan alrededor de USD\$18,8 mil millones anuales contribuyendo con una fuerza laboral de más de 10 millones de trabajadores. En el mundo la industria creativa es una importante impulsora del empleo generando en algunos países de Latinoamérica entre el $5 \%$ y el $11 \%$. Es importante destacar que el empleo generado por esta industria es mayoritariamente ocupado por jóvenes (GALLEGUILLOS ARAYASCHÜBELIN X. y ROBLES MARTíNEZ E. 2018 p.5).

Si bien, las cifras anteriores indicaron un crecimiento económico, como contrapartida atraer una determinada clase puede significar un desplazamiento de población, crecimiento del conflicto interno, problemas en la provisión de servicios públicos. Además que no se puede desconocer las prácticas de los habitantes, desjerarquizar trayectorias locales, la preexistencia de otros actores o la red de lazos sociales.

Por otro lado, el impulso de nuevas transformaciones que se adapten a las necesidades de esta "clase creativa" pueden afectar a la renta del suelo o la brecha de renta y la especulación sobre el mismo. Se considera la brecha de la renta a la "disparidad entre el nivel de renta del suelo potencial y la actual renta del suelo capitalizada bajo el presente uso de suelo" (ARREORTUA SALINAS, 2019 p.52), refiere a "la renta potencial que se puede obtener por cambio de uso de suelo" o la intensificación de 
su uso. Por ello, resulta de relevancia estudiar cómo evolucionan los valores del suelo urbano históricamente, si el valor es bajo los desarrollistas urbanos pueden estar interesados en comprar suelo porque eventualmente en un futuro, la ganancia sería extraordinaria.

Ahora bien, para comprender la dinámica de reproducción de suelo urbano en el barrio Güemes es necesario marcar los puntos de inflexión que sucedieron en la barrialidad desde sus orígenes. En el próximo apartado se mencionan aquellos episodios que marcaron y definieron al territorio.

\section{BREVE HISTORIA DEL BARRIO GÜEMES}

Barrio Güemes está ubicado hacia el sudeste del centro de la ciudad. surgió antes de 1870, fue una de las primeras ampliaciones del tejido sin planificación alguna (BOIXADÓS, 2000). Hacia principios del siglo XX, los habitantes identificaron cuatro sectores hacia el interior: El Abrojal; Pueblo Nuevo; La Bomba y El Infiernillo. El área de Pueblo Nuevo (parte del actual barrio Güemes) fue un punto de confluencia para los comerciantes que provenían de los caminos de La Rioja y Cuyo, por lo que representaba el eje transversal de la vida económica y social barrial.

En 1864 los vecinos solicitaron el establecimiento de una escuela y de una plaza, fue nominada por ordenanza municipal como "Plaza de las Carretas". La plaza funcionaba como lugar de carga, descarga, mercado de productos y parada de tropas provenientes de otras provincias (BISCHOFF, 1997). Hacia 1880 el barrio, fue incluido dentro del radio municipal, con la pretensión de modernizar la zona colindante al centro, extender y mejorar la calidad de los servicios. A finales de la década de 1880, durante la intendencia de Luis Revol se realizó el primer complejo de casas para obreros y población de clase baja que suponía grandes avances para la época y en el marco de las políticas higienistas a escala nacional.

Un siglo más tarde, en $1980^{3}$ se refuncionalizaron algunas de las casas del plan de Revol y la "Plaza de las Carretas" y se emplazó, una plaza seca actualmente conocida como "El Paseo de las Artes". Este, sería el espacio fundacional de las ferias de artesanos, que en un primer momento sólo contaba con 60 puestos y se convertiría con el transcurso de los años en la zona elegida de concurrencia.

A escala nacional, desde la década de los setenta el rol del Estado fue virando de un Estado "presente" del tipo keynesiano hacia uno del tipo competitivo en el marco del cambio del régimen de

\footnotetext{
${ }^{3}$ El Intendente en ese momento era el Teniente Gavier Olmedo y el encargo de la obra se realizó al arquitecto Miguel Ángel Roca.
} 
acumulación de capital. La nación Argentina se insertó en una nueva división internacional-regional del trabajo privilegió los espacios cuyas economías fueran susceptibles de amoldarse a las demandas del mercado global (MARENGO, 2010). Hacia los noventa asistió a la implementación de un paquete de medidas neoliberales (privatización, flexibilización laboral, desregulación, entre otras) que terminaron por romper la base del Estado benefactor. Esto se replicó en las provincias y municipios, en el caso de Córdoba se inauguró a comienzos de los noventa el surgimiento de ciudad competitiva a través de la implementación del primer Plan Estratégico (1991-1994). En la cual tenía un papel activo el sector privado en los debates de políticas públicas. El barrio Güemes fue una de las zonas elegidas para la ejecución de proyectos que tenían por objetivo: la activación económica, aplicar el modelo sustentable, generar un polo atractivo para actividades culturales y turísticas y la revitalización de cascos centrales y lugares patrimoniales.

El gobierno local implementó una serie de ordenanzas y planes, con el objetivo de poner en valor áreas que guardaban de alguna manera, una parte de la historia cordobesa y de reconstruir el sentido de la imagen de Córdoba patrimonial ${ }^{4}$, asociándose al pasado colonial. Esto fue consolidando la imagen de "barrio tradicional" ${ }^{5}$ de la ciudad. La nominación tiene fundamento en la primigenia localización fuera del centro de la ciudad y con cierta autonomía. También porque poseía algunos servicios urbanos: amojonamiento de parcelas, tendido eléctrico, tranvía, almacenes de ramos generales y más tarde recolección de residuos. Sin embargo, se constituyó como una extensión del centro fundacional y con una fuerte dependencia funcional de su centralidad.

Esta representación externa del barrio atrajo nuevos actores, hacia el 2000 comenzaron a abrir locales de variados rubros caracterizados por la imagen bohemia y artesanal. Además, la feria histórica creció en cantidad de artesanos y productos ofrecidos. Finalmente en el 2013 se inauguró la primera galería comercial, su diseño y construcción fue ejecutada bajo los lineamientos del modelo sustentable.

\footnotetext{
4 Planes y proyectos implementados desde 1980, algunos específicos en el barrio Güemes: 1- PLANDEMET (1980) -Plan de Desarrollo Metropolitano-, 2- PEC (1991-1999) -Plan Estratégico para la ciudad de Córdoba-; 3PECba (2003-2006)-El Plan Estratégico Córdoba-;4- "Portal Güemes”, hacia el año 2009 y El Plan Director 2008. Además, en el período 1995-1999 fueron abiertos anticuarios, lo cual permitió que el barrio Güemes sea caracterizado como zona para la compra-venta de esos objetos.

${ }^{5}$ Los barrios tradicionales son: barrio Güemes al suroeste junto con San Vicente y General Paz hacia el este, Alberdi hacia el oeste concordante con la direccionalidad del valle, Alta Córdoba al norte cruzando el Río Suquía, Nueva Córdoba al sur y Cerro de las Rosas al norte.
} 


\section{ASPECTOS METODOLÓGICOS \\ RESULTADOS \\ INTERVENCIÓN Y REESTRUCTURACIÓN ECONÓMICA ESPACIAL EN EL BARRIO}

En el periodo de tiempo analizado (2000-2016), la inversión y gestión empresarial del territorio permitió consolidar una lógica mercantil. Hacia el 2000 a escala global la renovación de espacios urbanos era tendencia. Por ello reaparece la idea de espacio 'obsoleto', 'peligroso', 'abandonado' que no cumple ninguna función. La planificación de la ciudad se vuelve una mercancía como atractor de inversiones (VAINER, 2000) fue el paradigma para producir 'nuevas áreas renovadas'.

Si bien el valor del suelo urbano aumentó exponencialmente, existen actores económicos con el suficiente capital interesados en desarrollar emprendimientos inmobiliarios varios, pues cada vez más se elige al barrio para emplazar un nuevo negocio o edificación en altura. Estos están dirigidos a cierta clase social, denominada nueva clase media o clase creativa (FLORIDA 2010). Los rasgos predominantes son el tipo de oferta de entretenimiento, cultural, artística, gastronómica y tiendas de diseño asociadas a un estilo de vida. Siguiendo a Arreortua Salinas (2019), este proceso ha sido posible por las ventajas derivadas de la brecha de renta: la ganancia potencial que se puede obtener en un determinado lugar (que será mayor que esa primera inversión) como consecuencia de los cambios de usos del suelo. En la (re)producción de espacio urbano-barrial priman intereses económicos.

En los últimos años, el paquete de políticas públicas que intervino en el barrio giró en torno a promover un proceso de renovación urbana edilicia manifestado con la construcción de edificios de distintas alturas, producto de por la Ordenanza 12483/15 que modificó sustancialmente la Ordenanza 8256/86 de Ocupación de suelo. La conjunción entre la modificación del corpus normativo, nuevas áreas de promoción urbana y la imagen patrimonial basada en el legado histórico abonaron el camino para la llegada de comercios.

El boom comercial, reconvirtió el uso de suelo, el espacio sufrió una reconfiguración en donde lo artesanal y bohemio y lo trendy parecen no tener un límite claro. El espacio barrial atiende el paseo con un recorrido completo: venta de productos, recorridos por la feria de tiempo libre y consumo en los bares-restaurantes. Los más de 600 artesanos emplazados en la plaza y en las calles adyacentes funcionan como un conector entre las calles peatonalizadas durante los fines de semana y una parte más del paseo. 


\section{CONCLUSIONES}

El caso expuesto del barrio Güemes mostró cómo el modo de reproducción de la lógica capitalista impacta en el desarrollo urbano local. Las transformaciones se inscriben como resultado de la planificación estratégica del espacio barrial, el foco de las acciones públicas mantuvo y profundizó las diferencias intrabarriales.

Las dos actualizaciones de valor del suelo urbano, una en el 2008 y otra en 2013, ambas impactaron en la contribución municipal por Inmuebles ${ }^{10}$. Si bien, no era el objetivo realizar un análisis del mercado de suelo urbano se reconoce que el valor de la tierra, incide en la producción y reproducción del espacio urbano. Estas múltiples intervenciones en la ciudad, alteran y transforman la estructura urbana, introducen cambios - por lo general en zonas centrales un aumento en los precios de los terrenos, y en los lotes desarrollados por el Estado, también en aquellos que se relacionan espacialmente con ello (JARAMILLO, 2009). El impacto de los valores del suelo, el cambio de la lógica residencial a comercial y la construcción de viviendas unifamiliares, se constituyen como elementos transformadores de determinado sector barrial. Ahora bien, el incremento de la brecha de renta fue posibilitado por los cambios normativos en el uso del suelo. Se alude que, de una función residencial se incorporaron incesantemente otras funciones: comerciales, de servicios, de esparcimiento y culturales y una mayor densidad residencial (viviendas colectivas, departamentos) asociado a una mayor rentabilidad.

Las políticas de renovación urbana promovidas por la gestión local en consonancia con inversores privados han desencadenado una dinamización de actividades, modificando el sentido tradicional del barrio especialmente en el sector colindante al centro histórico. Justamente fueron los procesos de renovación urbana, la puesta en valor del patrimonio, la promoción comercial y turística que modernizaron una centralidad barrial y afectaron profundamente al significado que esta área tenía en períodos anteriores.

Para futuras líneas de investigación queda el desafío por desarrollar los principales afectados por estas transformaciones y para quién realmente son destinados estos espacios barriales renovados. Debido a que el territorio es dinámico y aún se encuentra atravesado por varios proyectos de gestión local resta por conocer cómo estos planes incluirán las voces de los múltiples actores sociales que se encuentran en él.

\footnotetext{
${ }^{10}$ Según los planos municipales los datos fueron extraídos: "a partir de trabajos de valuación masiva con fines fiscales llevados adelante por la Dirección de Catastro Municipal entre mayo y diciembre de 2008."
} 


\section{REFERENCIAS BIBLIOGRÁFICAS}

ARREORTUA SALINAS, Luis. Transformaciones urbanas, procesos de gentrificación en ciudades latinoamericanas (p. 45-57). En: MARENGO, Cecilia et al. Crecimiento urbano: hacia una transformación sustentable del territorio. Compilado por María Cecilia Marengo. Córdoba: Editorial de la FAUD-UNC, 2019.

AYUNTAMIENTO DE BILBAO. Plan a Medio Plazo de la Asociación (1996-2000). Bilbao metrópoli, 1995.

BENJAMIN, Walter. Poesía y Capitalismo. Iluminaciones II. Madrid: Taurus, 1999.

BISCHOFF, Efraín. Historia de los Barrios de Córdoba. Sus leyendas, instituciones y gente Tomo I [1986]. Córdoba: Editorial Copiar, 1997.

BOITO, Eugenia y ESPOZ, María Belén. Urbanismo estratégico y separación clasista. Instantáneas de la ciudad en conflicto. Rosario, Argentina: Puño y Letra Editorialismo de Base, 2014.

BOIXADÓS, Cristina. Las Tramas de una ciudad, Córdoba entre 1870 y 1895. Élite urbanizadora, Infraestructura, poblamiento. Córdoba: Ferreyra editor, 2000.

BRITES, Walter. La ciudad en la encrucijada neoliberal. Urbanismo mercado-céntrico y desigualdad socio-espacial en América Latina. Urbe. Revista Brasileira de Gestão Urbana, 9(3), p. 573-586, 2017. https://doi.org/10.1590/2175-3369.009.003.ao14

CARMAN, María. El proceso de ennoblecimiento y la salida negociada de los innobles en Buenos Aires. Cadernos Metrópole, São Paulo, n.25 v. 13, p. 257-278, enero-junio, 2011. Recuperado de: https://www.redalyc.org/articulo.oa?id=402837820011

ENGELS, Friedrich. Contribución al problema de la vivienda. Leipzig: Volksstaat, 1873. Recuperado de: https://www.marxists.org/espanol/m-e/1870s/vivienda/

FALÚ Ana y MARENGO Cecilia. Strategic Planning in the city of Córdoba (p. 361 - 368). En:

Globalization, Urban form \& Governance. Delft: Delft University Press Science, 2002.

FLORIDA, R. La clase creativa. La transformación de la cultura del trabajo y del ocio en el siglo XXI. Madrid: Paidós, 2010.

GALLEGUILLOS ARAYA-SCHÜBELIN, Miriam Ximena y ROBLES MARTínEZ Elena. La creatividad como vector de desarrollo. Revista Pensum, v. 4, p. 1-16, 2018. Recuperado de:

https://revistas.unc.edu.ar/index.php/pensu/article/view/22605/22224

GLASS, Ruth. London: Aspects of Change. London: MacGibbon \& Kee, 1964.

GOTHAM, Kevin Fox. Tourism Gentrification: The Case of New Orleans' Vieux Carre (French Quarter). Urban Studies, n. 7, v. 42, p. 1099-1121, 2004.

HARVEY, David. Ciudades Rebeldes del Derecho de la ciudad a la revolución urbana. Salamanca: Editorial Akal, 2013.

HARVEY, David. The Right to the City, en Monthly Review. New Left Review, 53, p. 23-39, 2008. 
Recuperado de: http://newleftreview.es/?issue=53.

HARVEY, David. El Nuevo Imperialismo. Acumulación por desposesión. En Socialist Register Buenos Aires: CLACSO, 2004.

HERNÁNDEZ LÓPEZ, José. Tequila: Centro Mágico, Pueblo Tradicional. ¿Patrimonialización o Privatización?. Andamios, v. 12, n. 6, p. 41-67, 2009. Recuperado de:

https://www.redalyc.org/articulo.oa?id=628/62815957003

HERNÁNDEZ SAMPIERI, Roberto. et al. Metodología de la Investigación. México: Mc Graw Hill, 2006.

JACOBS, Jane. Muerte y vida de las grandes ciudades. Madrid: Península, 1973.

JARAMILLO GONZÁLEZ, S. Hacia una teoría de la renta del suelo urbano 2a edición- Bogotá:

Universidad de los Andes, Facultad de Economía, CEDE, Ediciones Uniandes, 2009.

LEFEBVRE, Henri. The production of space. (1974 Paris: Anthropos), (1991 Oxford: Basil Blackwell. Originally published 1974).

MARENGO, Cecilia. La planificación del crecimiento urbano: entre la regulación, la flexibilización normativa y las desigualdades socio-espaciales. Revista Científica Guillermo de Ockham, v.2, n. 8, p. 69-83, 2010. Recuperado de: https://www.redalyc.org/pdf/1053/105316833006.pdf

MÉNDEZ, Ricardo y CARAVACA, Inmaculada. Organización industrial y territorio. Capítulo 09 Cambio industrial en los espacios urbanos y rurales (p. 257-289). Madrid: Editorial Síntesis, 1996.

PECK, Jamie.(2012). Neoliberalismo y crisis actual. Documentos y Aportes en Administración Pública y Gestión Estatal, v.19, n. 12, p. 7-27, 2012. Recuperado de:

https://www.redalyc.org/pdf/3375/337530223001.pdf

QUEVEDO, Cecilia. Estados locales y alteridades indígenas. Sentidos sobre la inclusión habitacional en El Impenetrable. Córdoba: Editorial del CEA-FCS-UNC, 2018. Recuperado de:

https://rdu.unc.edu.ar/handle/11086/11767

SOJA, Edward. Postmetropolis: critical studies of cities and regions. Los Ángeles: Blackwell Publishing, 2000.

THEODORE, Nick; PECK, Jamie y BRENNER, Neil. Urbanismo neoliberal: la ciudad y EL IMPERIO de los mercados. Revista Temas Sociales, 66, 2009. Recuperado de: www.sitiosur.cl

VAINER, Carlos. Pátria, empresa e mercadoria. Notas sobre a estratégia discursiva do Planejamento Estratégico Urbano (p. 75-104). En: ARANTES Otilia, VAINER, Carlos y MARICATO, Erminia. A cidade do pensamento único. Desmanchando consensos. 3ra edición, Petropolis Río de Janeiro: editora Vozes, 2000.

WALLERSTEIN, Immanuel. Análisis de Sistemas-mundo: Una introducción. Madrid: Siglo XXI, 2005.

ZUKIN, Sharon. Paisagens urbanas pós-modernas: mapeando cultura e poder. Revista do Patrimonio Historico e Artistico Nacional, n. 24, p. 205- 219, 1996. Cidadania (Curadoria Antonio Arantes). Recuperado de: https://csociais.files.wordpress.com/2018/09/zukin-paisagens-urbanaposmodernas-mapeando-cultura-e-poder.pdf 
ZUKIN, Sharon. Naked City: the death and life of authentic urban places. Oxford, UK; New York : Oxford University Press, 2010.

\section{ORDENANZAS MUNICIPALES}

Ordenanza 8057, 1985. Ocupación del Suelo y Preservación de Ámbitos Históricos, Arquitectónicos y Paisajísticos dentro del Área Central de la Ciudad. Concejo Deliberante, Municipalidad de Córdoba.

Ordenanza 8256, 1986. Ocupación del Suelo. Concejo Deliberante, Municipalidad de Córdoba.

Ordenanza 11202, 2007. Establece acciones de tutela de valores culturales/paisajísticos de bienes componentes del Patrimonio. Concejo Deliberante, Municipalidad de Córdoba.

Ordenanza 12201, 2013. Reemplaza Catálogo de Bienes Inmuebles y Lugares del Patrimonio Cordobés. Concejo Deliberante, Municipalidad de Córdoba.

Ordenanza 12.483, 2015. Uso y Ocupación del Suelo. Concejo Deliberante, Municipalidad de Córdoba. 